\title{
The Solution of Initial Boundary Value Problem with Time and Space-Fractional Diffusion Equation via a Novel Inner Product
}

\author{
Hulya Kodal Sevindir (D) and Ali Demir \\ Kocaeli University, Department of Mathematics, 41380 Kocaeli, Turkey \\ Correspondence should be addressed to Hulya Kodal Sevindir; hulyasevindir@gmail.com
}

Received 20 February 2018; Accepted 6 May 2018; Published 6 June 2018

Academic Editor: Giorgio Kaniadakis

Copyright (C) 2018 Hulya Kodal Sevindir and Ali Demir. This is an open access article distributed under the Creative Commons Attribution License, which permits unrestricted use, distribution, and reproduction in any medium, provided the original work is properly cited.

\begin{abstract}
The main goal of this study is to find the solution of initial boundary value problem for the one-dimensional time and spacefractional diffusion equation which is a very intriguing topic for many researchers. With the aim of newly defined inner product, which is the main contribution of this study, the analytic solution of the boundary value problem is obtained. The time and spacefractional derivatives are defined in the Caputo sense which is more suitable than Riemann-Liouville sense. We apply the separation of variables method to reduce the problem to two separate fractional ODEs. The generalized solution is constructed/formed in the form of a Fourier series with respect to the eigenfunctions of a certain eigenvalue problem. In order to obtain the coefficients of the Fourier series for the solution, we define a new inner product which is the key point of study.
\end{abstract}

\section{Introduction}

Since PDE of fractional order contributes to modeling for the wide range of processes and systems, including past memories, in various scientific research areas, it has become very intriguing topic for many scientists. Main reason of this trend is that using fractional derivative is global in nature whereas the integer derivative is local in nature. This property makes fractional DEs the best possible choice in modeling physical problems involving past memory and/or delay and attracts growing number of researchers.

The Caputo derivatives are more useful than RiemannLiouville derivatives since the analysis of the mathematical models involving Caputo derivatives gives closer results to the analysis of ones including integer derivatives. There are various studies in literature supporting this conclusion [18]. By making use of Mittag-Leffler function, characteristic equations of fractional ODEs are solved and solutions of them are constructed efficiently. In this sense, the Mittag-Leffler function takes the role of the exponential function which is used in the determination of solutions for ODEs with integer derivatives.

\section{Preliminary Results}

In this section, some basic definitions and known results regarding Caputo fractional derivatives are given.

Definition 1. The Caputo fractional derivative of $u(t)$ of order $q$ where $n-1<q<n$ is given by equation

$$
\begin{aligned}
{ }^{c} D^{q} u(t)=\frac{1}{\Gamma(\mathrm{n}-\mathrm{q})} \int_{t_{0}}^{t}(t-s)^{n-q-1} u^{(n)}(s) d s, & \\
& t \in\left[t_{0}, t_{0}+T\right]
\end{aligned}
$$

where $u^{(n)}(t)=d^{n} u / d t^{n}$. If $q$ is an integer, then the above definition of fractional derivative coincides with the integer derivative.

Definition 2. If $0<q<1$, the Caputo fractional derivative of order $q$ can be defined by equation

$$
{ }^{c} D^{q} u(t)=\frac{1}{\Gamma(1-\mathrm{q})} \int_{t_{0}}^{t}(t-s)^{-q} u^{\prime}(s) d s
$$

where $0<\mathrm{q}<1$. 
To determine the solution of eigenvalue problem in the following section, we use the two-parameter Mittag-Leffler function defined as

$$
E_{\alpha, \beta}\left(\lambda\left(t-t_{0}\right)^{\alpha}\right)=\sum_{k=0}^{\infty} \frac{\left(\lambda\left(t-t_{0}\right)^{\alpha}\right)^{k}}{\Gamma(\alpha k+\beta)}, \quad \alpha, \beta>0
$$

where $\lambda$ is a constant. In particular, for $t_{0}=0, \alpha=\beta=q$ we obtain

$$
E_{q, q}\left(\lambda t^{q}\right)=\sum_{k=0}^{\infty} \frac{\left(\lambda t^{q}\right)^{k}}{\Gamma(q k+q)}, \quad q>0
$$

When $q=1$, Mittag-Leffler function corresponds to usual exponential function, i.e., $E_{1,1}(\lambda t)=e^{\lambda t}$. For further reading see $[9,10]$.

Let us define the following functions which play an important role in the representation of solution for the problem under consideration:

$$
\begin{aligned}
\sin _{q}\left(\mu t^{q}\right) & =\frac{E_{q, 1}\left(i \mu t^{q}\right)-E_{q, 1}\left(-i \mu t^{q}\right)}{2 i} \\
& =\sum_{k=0}^{\infty} \frac{(-1)^{k}\left(\mu t^{q}\right)^{2 k+1}}{\Gamma((2 k+1) q+1)}
\end{aligned}
$$

and

$$
\begin{aligned}
\cos _{q}\left(\mu t^{q}\right) & =\frac{E_{q, 1}\left(i \mu t^{q}\right)+E_{q, 1}\left(-i \mu t^{q}\right)}{2} \\
& =\sum_{k=0}^{\infty} \frac{(-1)^{k}\left(\mu t^{q}\right)^{2 k}}{\Gamma(2 k q+1)} .
\end{aligned}
$$

Note that for $q=1$ these functions are usual trigonometric functions $\sin (\mu t)$ and $\cos (\mu t)$.

\section{Novel Inner Product}

In this section, we define a new inner product to determine the solution which satisfies the initial boundary value problem. The missing of such an inner product makes the space-time fractional initial and boundary value problems open problems. This inner product promotes these kinds of problems to be studied theoretically. Moreover, it provides the best understanding possible to develop the theoretical study of space-time fractional initial boundary value problems. By this inner product, the maximum principle for time-spacefractional PDE will be discussed and investigated for a wide range of problems. In the definition of this inner product, we use the functions which satisfy the initial and boundary value problems with integer orders which make the calculations much more easier.

Theorem 3. Let $V$ be a vector space, made up of all linear combinations of $\sin _{\beta}\left(\mu(x /(b-a))^{\beta}\right)$ and $\cos _{\beta}\left(\mu(x /(b-a))^{\beta}\right)$ for fixed $\beta$ where $0<\beta \leq 1, \mu \in \mathbb{R}$ on the interval $I=[a, b]$, i.e., $V=\operatorname{span}\left\{\sin _{\beta}\left(\mu(x /(b-a))^{\beta}\right), \cos _{\beta}\left(\mu(x /(b-a))^{\beta}\right)\right\}$.
Let $T: V \rightarrow \operatorname{span}\{\sin (\mu x /(b-a)), \cos (\mu x /(b-a))\}$ be a linear transformation which is one-to-one and onto. Hence it has its inverse transformation $T^{-1}$. The mapping $<\cdot, \cdot>: V \times V \rightarrow \mathbb{R}$ is defined as

$$
\begin{aligned}
& \langle u(x ; \beta), v(x ; \beta)\rangle \\
& \quad=\left.T^{-1}\left(\int T u(x ; \beta) . T v(x ; \beta) d x\right)\right|_{x=a} ^{b}
\end{aligned}
$$

where $\operatorname{Tu}(x ; \beta)=u(x ; 1)$ and $\operatorname{Tv}(x ; \beta)=v(x ; 1)$. In other words,

$$
\langle u(x ; \beta), v(x ; \beta)\rangle=\int u(x ; 1) . v(x ; 1) d x=w(x, 1) .
$$

At this point we transform the function $w(x, 1)$ to the corresponding function $w(x, \beta)$. Replacing $\beta$ back and substituting upper and lower values give us the result of the new inner product.

$$
\begin{aligned}
\langle u(x ; \beta), v(x ; \beta)\rangle & =\left.w(x, \beta)\right|_{x=a} ^{x=b} \\
& =w(b, \beta)-w(a, \beta) .
\end{aligned}
$$

Proof. Let us show that this mapping satisfies the conditions of an inner product:

$$
\begin{aligned}
(1) & <u(x ; \beta), u(x ; \beta)>\geq 0 \\
& <u(x ; \beta), u(x ; \beta)>=\int u^{2}(x ; 1) d x=w(x, 1) \\
& <u(x ; \beta), u(x ; \beta)>=\left.w(x, \beta)\right|_{x=a} ^{x=b}=w(b, \beta)- \\
& w(a, \beta) \geq 0
\end{aligned}
$$

since $w(b, 1)-w(a, 1) \geq 0$ and $-1 \leq \sin _{q}(x) \leq 1,-1 \leq$ $\cos _{q}(x) \leq 1, \forall q \in(0,1)$.

Moreover $<u(x ; \beta), u(x ; \beta)>=0 \Leftrightarrow u(x ; \beta)=0$.

$(2)<u(x ; \beta), v(x ; \beta)>=\int u(x ; 1) v(x ; 1) d x=$ $\int v(x ; 1) u(x ; 1) d x=w(x, 1)$

$<u(x ; \beta), v(x ; \beta)>=\left.w(x, \beta)\right|_{x=a} ^{x=b}=w(b, \beta)-$ $w(a, \beta)=\langle v(x ; \beta), u(x ; \beta)\rangle$.

$(3)<u(x ; \beta)+v(x ; \beta), z(x ; \beta)>=\int(u(x ; 1)+$ $v(x ; 1)) z(x ; 1) d x$

$=\int u(x ; 1) z(x ; 1) d x+\int v(x ; 1) z(x ; 1) d x$

$<u(x ; \beta)+v(x ; \beta), z(x ; \beta)>=\left.w_{1}(x, \beta)\right|_{x=a} ^{b}+$ $\left.w_{2}(x, \beta)\right|_{x=a} ^{b}$

$=w_{1}(b, \beta)-w_{1}(a, \beta)+w_{2}(b, \beta)-w_{2}(a, \beta)$

$=\langle u(x ; \beta), z(x ; \beta)\rangle+\langle v(x ; \beta), z(x ; \beta)\rangle$.

$(4)<\alpha u(x ; \beta), v(x ; \beta)>=\int \alpha u(x ; 1) v(x ; 1) d x=$ $\alpha \int u(x ; 1) v(x ; 1) d x=\alpha w(x, 1)$

$<\alpha u(x ; \beta), v(x ; \beta)>=\left.\alpha w(x, \beta)\right|_{x=a} ^{b}=\alpha(w(b, \beta)-$ $w(a, \beta))=\alpha<u(x ; \beta), v(x ; \beta)>$. 
Example 4. Let us illustrate this inner product by an example in which we take the inner product of Mittag-Leffler functions $E_{\beta}\left(\lambda_{1}(\beta) x^{\beta}\right)$ and $E_{\beta}\left(\lambda_{2}(\beta) x^{\beta}\right)$ :

$$
\begin{aligned}
& \left\langle E_{\beta}\left(\lambda_{1}(\beta) x^{\beta}\right), E_{\beta}\left(\lambda_{2}(\beta) x^{\beta}\right)\right\rangle \\
& =\int e^{\lambda_{1}(1) x} e^{\lambda_{2}(1) x} d x=\int e^{\left(\lambda_{1}(1)+\lambda_{2}(1)\right) x} d x \\
& =\frac{e^{\left(\lambda_{1}(1)+\lambda_{2}(1)\right) x}}{\lambda_{1}(1)+\lambda_{2}(1)} \\
& \left\langle E_{\beta}\left(\lambda_{1}(\beta) x^{\beta}\right), E_{\beta}\left(\lambda_{2}(\beta) x^{\beta}\right)\right\rangle \\
& =\left.\frac{E_{\beta}\left(\lambda_{1}(\beta)+\lambda_{2}(\beta) x^{\beta}\right)}{\lambda_{1}(\beta)+\lambda_{2}(\beta)}\right|_{x=a} ^{b} \\
& =\frac{E_{\beta}\left(\lambda_{1}(\beta)+\lambda_{2}(\beta) b^{\beta}\right)-E_{\beta}\left(\lambda_{1}(\beta)+\lambda_{2}(\beta) a^{\beta}\right)}{\lambda_{1}(\beta)+\lambda_{2}(\beta)}
\end{aligned}
$$

Note that this inner product extends orthogonality property between the functions $u(x ; 1)$ and $v(x ; 1)$ to the functions $u(x ; \beta)$ and $v(x ; \beta)$.

\section{Main Results}

In this section, we deal with the following initial boundary value problem involving time and space-fractional PDE:

$$
\begin{aligned}
D_{t}^{\alpha} u(x, t) & =a D_{x}^{2 \beta}, \quad 0<\alpha<1,1<2 \beta<2, a>0 \\
u(x, 0) & =u_{o}(x), \quad 0 \leq x \leq l \\
u(0, t) & =0 \\
u(l, t) & =0
\end{aligned}
$$

$$
0 \leq t \leq T
$$

The generalized solution of this problem can be obtained in an analytical form by using the separation of variables method. Let a particular solution of (11)-(13) be in the following form:

$$
u(x, t ; \alpha, \beta)=X(x ; \beta) T(t ; \alpha, \beta)
$$

where $0 \leq x \leq l, 0 \leq t \leq T$.

Note that the function $X$ depends on order of fractional derivative with respect to $x$ and function $T$ depends on orders of fractional derivatives with respect to $x$ and $t$. Substituting (14) into (11) and applying separation of the variables lead to the equation

$$
\frac{\left(D^{\alpha} T\right)(t ; \alpha, \beta)}{a T(t ; \alpha, \beta)}=\frac{\left(D^{2 \beta} X\right)(x ; \beta)}{X(x ; \beta)}=-\lambda(\beta)
$$

where $\lambda$ depends on order of the fractional derivative with respect to $x$. The equations in (15) reduce the problem to two separate ODEs including fractional derivatives with respect to both time and space. The equation on the right with boundary conditions (13) gives the following fractional differential equation:

$$
\begin{aligned}
\left(D^{2 \beta} X\right)(x ; \beta)+\lambda(\beta) X(x ; \beta) & =0 \\
X(0 ; \beta) & =0, \quad X(l ; \beta)=0
\end{aligned}
$$

In order to determine the solution of the eigenvalue problem ((16)-(17)) we use the following Mittag-Leffler function:

$$
X(x ; \beta)=E_{\beta, 1}\left(r x^{\beta}\right)
$$

which leads to the following characteristic equation for the eigenvalue problem ((16)-(17)):

$$
r^{2}+\lambda(\beta)=0
$$

Case 1. If $\lambda<0$, we have two real and distinct solutions $r=$ $\pm \sqrt{-\lambda(\beta)}$, and the general solution of the eigenvalue problem ((16)-(17)) is represented by

$$
\begin{aligned}
X(x ; \beta) & =c_{1} E_{\beta, 1}\left(\sqrt{-\lambda} x^{\beta}\right)+c_{2} E_{\beta, 1}\left(-\sqrt{-\lambda} x^{\beta}\right) \\
X(0 ; \beta) & =0 \Longrightarrow c_{1} E_{\beta, 1}(0)+c_{2} E_{\beta, 1}(0)=0 \Longrightarrow c_{2} \\
& =-c_{1}
\end{aligned}
$$

which leads to the following solution:

$$
X(x ; \beta)=c_{1}\left(E_{\beta, 1}\left(\sqrt{-\lambda} x^{\beta}\right)-E_{\beta, 1}\left(-\sqrt{-\lambda} x^{\beta}\right)\right) .
$$

From the second boundary condition we have

$$
\begin{aligned}
X(l ; \beta) & =0 \Longrightarrow X(l ; \beta) \\
& =c_{1}\left(E_{\beta, 1}\left(\sqrt{-\lambda} l^{\beta}\right)-E_{\beta, 1}\left(-\sqrt{-\lambda} l^{\beta}\right)\right)=0
\end{aligned}
$$

which implies that

$$
E_{\beta, 1}\left(\sqrt{-\lambda} l^{\beta}\right)=E_{\beta, 1}\left(-\sqrt{-\lambda} l^{\beta}\right) .
$$

Thus

$$
\begin{aligned}
\sqrt{-\lambda} l^{\beta} & =0 \Longrightarrow \\
\lambda & =0 \\
X(x ; \beta) & =c_{1}\left(E_{\beta, 1}(0)-E_{\beta, 1}(0)\right)=0
\end{aligned}
$$

which means that there is no solution for the case $\lambda<0$.

Case 2. If $\lambda=0$, the characteristic equation becomes

$$
\begin{gathered}
r^{2}=0 \Longrightarrow \\
r_{1,2}=0 .
\end{gathered}
$$

Hence the general solution of the eigenvalue problem ((16)(17)) is represented by

$$
X(x ; \beta)=c_{1}+c_{2} \frac{x^{\beta}}{\beta}
$$


From the first boundary condition we have

$$
\begin{gathered}
X(0 ; \beta)=0 \Longrightarrow \\
c_{1}=0
\end{gathered}
$$

which leads to the following solution:

$$
X(x ; \beta)=c_{2} \frac{x^{\beta}}{\beta} .
$$

From the last boundary condition we have

$$
\begin{aligned}
X(l ; \beta) & =0 \Longrightarrow \\
c_{2} \frac{x^{\beta}}{\beta} & =0 \Longrightarrow \\
c_{2} & =0
\end{aligned}
$$

which leads to $X(x ; \beta)=0$ which means that there is no solution for $\lambda=0$.

Case 3. If $\lambda>0$, we obtain two complex roots $r=\mp i \sqrt{\lambda}$. Then the general solution of the eigenvalue problem $((16)-(17))$ is represented by

$$
X(x ; \beta)=c_{1} \sin _{\beta}\left(\sqrt{\lambda} x^{\beta}\right)+c_{2} \cos _{\beta}\left(\sqrt{\lambda} x^{\beta}\right) .
$$

Let us check if the boundary conditions are satisfied by the solution. From the first boundary condition we have

$$
\begin{aligned}
X(0 ; \beta) & =0 \Longrightarrow \\
c_{1} \sin _{\beta}(0)+c_{2} \cos _{\beta}(0) & =0 \Longrightarrow \\
c_{2} & =0
\end{aligned}
$$

which gives the following solution:

$$
X(x ; \beta)=c_{1} \sin _{\beta}\left(\sqrt{\lambda} x^{\beta}\right)
$$

From the second boundary condition we have

$$
\begin{aligned}
X(l ; \beta) & =0 \Longrightarrow \\
X(l) & =c_{1} \sin _{\beta}\left(\sqrt{\lambda} l^{\beta}\right)=0
\end{aligned}
$$

which implies that

$$
\sin _{\beta}\left(\sqrt{\lambda} l^{\beta}\right)=0
$$

Let $w_{n}(\beta)=\sqrt{\lambda_{n}(\beta)} l^{\beta}$. Hence the eigenvalues can be represented in terms of $w_{n}(\beta)$ as follows:

$$
\lambda_{n}(\beta)=\frac{w_{n}^{2}(\beta)}{l^{2 \beta}}, \quad 0<w_{1}(\beta)<w_{2}(\beta)<\cdots
$$

Thus the solution of the eigenvalue problem is represented in the following form:

$$
X_{n}(x ; \beta)=c_{n} \sin _{\beta}\left(w_{n}(\beta)\left(\frac{x}{l}\right)^{\beta}\right) .
$$

The equation on the left of (15) for each eigenvalue $\lambda_{n}$ gives the following fractional differential equation:

$$
\left(D^{\alpha} T_{n}\right)(t ; \alpha, \beta)+\left(\frac{a w_{n}^{2}(\beta)}{l^{2 \beta}}\right) T_{n}(t ; \alpha, \beta)=0
$$

By using the similar calculations the solution of (37) is determined in the following form:

$$
T_{n}(t ; \alpha, \beta)=c_{n} E_{\alpha}\left(-\left(\frac{a w_{n}^{2}(\beta)}{l^{2} \beta}\right) t^{\alpha}\right) .
$$

For each eigenvalue $\lambda_{n}$ we obtain the following function:

$$
\begin{aligned}
& u_{n}(x, t ; \alpha, \beta)=X_{n}(x ; \beta) T_{n}(t ; \alpha, \beta) \\
& \quad=c_{n} \sin _{\beta}\left(w_{n}(\beta)\left(\frac{x}{l}\right)^{\beta}\right) E_{\alpha}\left(-\left(\frac{a w_{n}^{2}(\beta)}{l^{2} \beta}\right) t^{\alpha}\right)
\end{aligned}
$$

and hence we have the following sum:

$$
\begin{aligned}
& u(x, t ; \alpha, \beta)=\sum_{n=1}^{\infty} u_{n}(x, t ; \alpha, \beta) \\
& =\sum_{n=1}^{\infty} c_{n} \sin _{\beta}\left(w_{n}(\beta)\left(\frac{x}{l}\right)^{\beta}\right) E_{\alpha}\left(-\left(\frac{a w_{n}^{2}(\beta)}{l^{2 \beta}}\right) t^{\alpha}\right)
\end{aligned}
$$

which satisfy both the fractional equation (11) and boundary condition (13).

In order to determine the solution which satisfies the initial condition (12) we use the inner product defined in (7).

In (38), replacing $t$ by 0 and using the initial condition (12) we have

$$
\begin{aligned}
u(x, 0 ; \alpha, \beta) & =u_{0}(x ; \alpha, \beta)=\sum_{n=1}^{\infty} u_{n}(x, 0 ; \alpha, \beta) \\
& =\sum_{n=1}^{\infty} c_{n} \sin _{\beta}\left(w_{n}(\beta)\left(\frac{x}{l}\right)^{\beta}\right) .
\end{aligned}
$$

Via the inner product (7) we will obtain the coefficients $c_{n}$ for $n=1,2,3, \ldots$ as follows:

$$
\begin{aligned}
c_{n}=\left\langle u_{0}(x ; \alpha, \beta), \sin _{\beta}\left(w_{n}(\beta)\left(\frac{x}{l}\right)^{\beta}\right)\right\rangle & \\
n & =1,2,3, \ldots
\end{aligned}
$$

\section{Illustrative Example}

Before solving fractional heat-like problem we first solve the following problem for $\alpha=\beta=1$ in order to compare the solutions of these two problems:

$$
\begin{aligned}
D_{t} u(x, t) & =\frac{1}{4} D_{x}^{2} u(x, t), \quad 0 \leq x \leq 2, t>0 \\
u(x, 0) & =-\sin (\pi x), \quad 0 \leq x \leq 2 \\
u(0, t) & =0 \\
u(2, t) & =0
\end{aligned}
$$

$$
0 \leq t \leq T
$$


The solution of this problem is obtained as follows:

$$
u(x, t)=-\sin (\pi x) \exp \left(\frac{-\pi^{2}}{4} t\right) .
$$

Let us consider the following fractional heat-like problem:

$$
\begin{aligned}
D_{t}^{\alpha} u(x, t) & =\frac{1}{4} D_{x}^{2 \beta} u(x, t), \quad 0<\alpha<1, \quad 1<2 \beta<2 \\
u(x, 0) & =-\sin (\pi x), \quad 0 \leq x \leq 2 \\
u(0, t) & =0 \\
u(2, t) & =0
\end{aligned}
$$

$$
0 \leq t \leq T
$$

Applying separation of the variables to (45) leads to the equation

$$
\frac{4\left(D^{\alpha} T\right)(t ; \alpha, \beta)}{T(t ; \alpha, \beta)}=\frac{\left(D^{2 \beta} X\right)(x ; \beta)}{X(x ; \beta)}=-\lambda(\beta)
$$

where $\lambda$ depends on $\beta$. Hence we get two ODEs having fractional derivatives with respect to time and space separately. The equation on the right of (48) with boundary conditions (47) gives the fractional differential equation

$$
\begin{aligned}
\left(D^{2 \beta} X\right)(x ; \beta)+\lambda X(x ; \beta) & =0 \\
X(0 ; \beta) & =0, \\
X(2 ; \beta) & =0 .
\end{aligned}
$$

Using the Mittag-Leffler function $X(x ; \beta)=E_{\beta, 1}\left(r x^{\beta}\right)$ we obtain the following characteristic equation $r^{2}+\mu^{2}(\beta)=0$ where we take $\lambda=\mu^{2}$. Hence the solution becomes

$$
X(x ; \beta)=c_{1} \sin _{\beta}\left(\mu x^{\beta}\right)+c_{2} \cos _{\beta}\left(\mu x^{\beta}\right) .
$$

Using boundary conditions

$$
\begin{aligned}
X(0 ; \beta) & =0 \Longrightarrow \\
c_{1} \sin _{\beta}(0)+c_{2} \cos _{\beta}(0) & =0 \Longrightarrow \\
c_{2} & =0
\end{aligned}
$$

which gives the following solution:

$$
X(x ; \beta)=c_{1} \sin _{\beta}\left(\mu x^{\beta}\right) .
$$

From the second boundary condition we have

$$
\begin{aligned}
& X(2 ; \beta)=0 \Longrightarrow \\
& X(2 ; \beta)=c_{1} \sin _{\beta}\left(\mu 2^{\beta}\right)=0
\end{aligned}
$$

which implies that

$$
\sin _{\beta}\left(\mu 2^{\beta}\right)=0
$$

Let $w_{n}(\beta)=\mu_{n} 2^{\beta}$, i.e., $\sin _{\beta}\left(w_{n}\right)=0$. Hence the eigenvalues can be represented in terms of $w_{n}(\beta)$ as follows:

$$
\lambda_{n}(\beta)=\frac{w_{n}^{2}(\beta)}{2^{2 \beta}}, \quad 0<w_{1}(\beta)<w_{2}(\beta)<\cdots
$$

Thus the solution of the eigenvalue problem is represented in the following form:

$$
X_{n}(x ; \beta)=c_{n} \sin _{\beta}\left(w_{n}(\beta)\left(\frac{x}{2}\right)^{\beta}\right) .
$$

The equation on the left of (48) for each eigenvalue $\lambda_{n}(\beta)$ gives the following fractional differential equation:

$$
\left(D^{\alpha} T_{n}\right)(t ; \alpha, \beta)+\left(\frac{w_{n}^{2}(\beta)}{2^{2 \beta+2}}\right) T_{n}(t ; \alpha, \beta)=0 .
$$

By using the similar calculations the solution of (58) is determined in the following form:

$$
T_{n}(t ; \alpha, \beta)=E_{\alpha}\left(-\left(\frac{w_{n}(\beta)}{2^{\beta+1}}\right)^{2} t^{\alpha}\right) .
$$

For each eigenvalue $\lambda_{n}$ we obtain the following function:

$$
\begin{aligned}
& u_{n}(x, t ; \alpha, \beta)=X_{n}(x ; \beta) T_{n}(t ; \alpha, \beta) \\
& \quad=c_{n} \sin _{\beta}\left(w_{n}(\beta)\left(\frac{x}{2}\right)^{\beta}\right) E_{\alpha}\left(-\left(\frac{w_{n}(\beta)}{2^{\beta+1}}\right)^{2} t^{\alpha}\right)
\end{aligned}
$$

and thus we have the following sum:

$$
\begin{aligned}
& u(x, t ; \alpha, \beta)=\sum_{n=1}^{\infty} u_{n}(x, t ; \alpha, \beta) \\
& \quad=\sum_{n=1}^{\infty} c_{n} \sin _{\beta}\left(w_{n}(\beta)\left(\frac{x}{2}\right)^{\beta}\right) E_{\alpha}\left(-\left(\frac{w_{n}(\beta)}{2^{\beta+1}}\right)^{2} t^{\alpha}\right)
\end{aligned}
$$

which satisfy both (45) and boundary condition (47). In order to determine the solution which satisfies the initial condition (46),

$$
\begin{aligned}
u(x, 0 ; \alpha, \beta) & =\sum_{n=1}^{\infty} u_{n}(x, 0 ; \alpha, \beta) \\
& =\sum_{n=1}^{\infty} c_{n} \sin _{\beta}\left(w_{n}(\beta)\left(\frac{x}{2}\right)^{\beta}\right) \\
& =-\sin (\pi x) .
\end{aligned}
$$

Via the inner product we obtain the coefficients $c_{n}$ for $n=$ $1,2,3, \ldots$ as follows:

$$
c_{n}=\left\langle-\sin (\pi x), \sin _{\beta}\left(w_{n}(\beta)\left(\frac{x}{2}\right)^{\beta}\right)\right\rangle,
$$




$$
\begin{aligned}
c_{n}= & -\int \sin (\pi x) \sin \left(\frac{n \pi x}{2}\right) d x, \quad n \neq 2 \\
= & \frac{1}{(n-2) \pi} \sin \left(\frac{(n-2) \pi x}{2}\right) \\
& -\frac{1}{(n+2) \pi} \sin \left(\frac{(n+2) \pi x}{2}\right) .
\end{aligned}
$$

Replacing $\beta$ back and substituting upper and lower limits give us the result of the new inner product.

$$
\begin{aligned}
c_{n}= & \frac{1}{w_{n-2}} \sin _{\beta}\left(w_{n-2}(\beta)\left(\frac{x}{2}\right)^{\beta}\right) \\
& -\left.\frac{1}{w_{n+2}} \sin _{\beta}\left(w_{n+2}(\beta)\left(\frac{x}{2}\right)^{\beta}\right)\right|_{x=0} ^{x=2} \\
= & \frac{1}{w_{n-2}} \sin _{\beta}\left(w_{n-2}(\beta)\right)-\frac{1}{w_{n+2}} \sin _{\beta}\left(w_{n+2}(\beta)\right)
\end{aligned}
$$

Thus $c_{n}=0, n \neq 2$.

For $n=2$ we get

$$
\begin{aligned}
c_{2} & =\left\langle-\sin (\pi x), \sin _{\beta}\left(w_{2}(\beta)\left(\frac{x}{2}\right)^{\beta}\right)\right\rangle, \\
& n=1,2,3, \ldots \\
& =-\int \sin ^{2}(\pi x) d x=-\frac{1}{2}\left(x+\frac{\sin 2 \pi x}{4 \pi}\right) \\
& =-\frac{1}{2} x^{\beta}+\left.\frac{\sin _{\beta}\left(w_{4}(\beta)(x / 2)^{\beta}\right)}{w_{4}(\beta)}\right|_{x=0} ^{x=2}=-2^{\beta-1} .
\end{aligned}
$$

Thus

$$
\begin{gathered}
u(x, t ; \alpha, \beta)=\sum_{n=1}^{\infty} u_{n}(x, t ; \alpha, \beta) \\
=-2^{\beta-1} \sin _{\beta}\left(w_{2}(\beta)\left(\frac{x}{2}\right)^{\beta}\right) \\
\cdot E_{\alpha}\left(-\left(\frac{w_{2}(\beta)}{2^{\beta+1}}\right)^{2} t^{\alpha}\right) .
\end{gathered}
$$

Notice that as $\beta$ and $\alpha$ go to 1 , solution (67) goes to the solution (44).

\section{Conclusion}

In this paper, we establish a new inner product which plays the remarkable role in the determination of the solution for the one-dimensional time and space-fractional diffusion problem which is an open problem. Moreover this inner product can be applied in many related problems and brings new insights into the understanding of time and spacefractional problems.

\section{Data Availability}

No data were used to support this study.

\section{Conflicts of Interest}

The authors declare that there are no conflicts of interest regarding the publication of this article.

\section{Authors' Contributions}

All authors read and approved the final manuscript.

\section{References}

[1] A. Demir, S. Erman, B. Özgür, and E. Korkmaz, "Analysis of fractional partial differential equations by Taylor series expansion," Boundary Value Problems, vol. 68, 2013.

[2] A. Demir, F. Kanca, and E. Ozbilge, "Numerical solution and distinguishability in time fractional parabolic equation," Boundary Value Problems, vol. 142, 2015.

[3] E. Ozbilge and A. Demir, "Analysis of the inverse problem in a time fractional parabolic equation with mixed boundary conditions," Boundary Value Problems, vol. 134, 2014.

[4] F. Huang and F. Liu, "The time fractional diffusion equation and the advection-dispersion equation," The ANZIAM Journal, vol. 46, no. 3, pp. 317-330, 2005.

[5] Y. Luchko, "Initial-boundary-value problems for the onedimensional time-fractional diffusion equation," Fractional Calculus and Applied Analysis An International Journal for Theory and Applications, vol. 15, no. 1, pp. 141-160, 2012.

[6] Y. Luchko, "Initial-boundary problems for the generalized multi-term time-fractional diffusion equation," Journal of Mathematical Analysis and Applications, vol. 374, no. 2, pp. 538548, 2011.

[7] S. Momani and Z. Odibat, "Numerical comparison of methods for solving linear differential equations of fractional order," Chaos, Solitons and Fractals, vol. 31, no. 5, pp. 1248-1255, 2007.

[8] L. Plociniczak, "Analytical studies of a time-fractional porous medium equation. Derivation, approximation and applications," Communications in Nonlinear Science and Numerical Simulation, vol. 24, no. 1, pp. 169-183, 2015.

[9] A. A. Kilbas, H. M. Srivastava, and J. J. Trujillo, Theory and Applications of Fractional Differential Equations, Elsevier, Amsterdam, 2006.

[10] I. Podlubny, Fractional Differential Equations, Academic Press, San Diego, Calif, USA, 1999. 


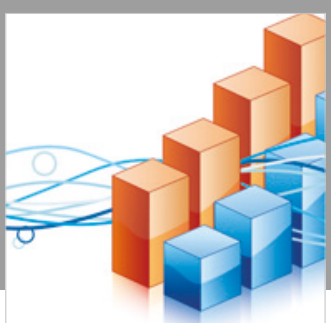

Advances in

Operations Research

\section{-n-m}
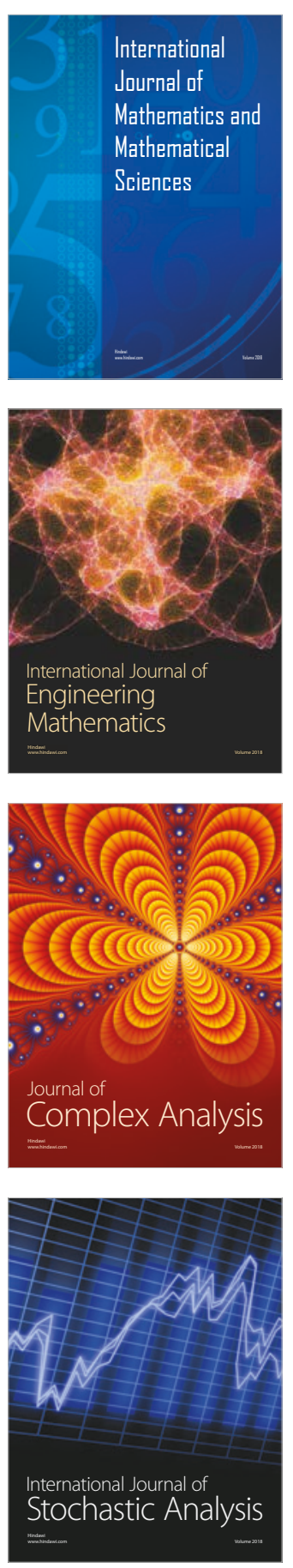
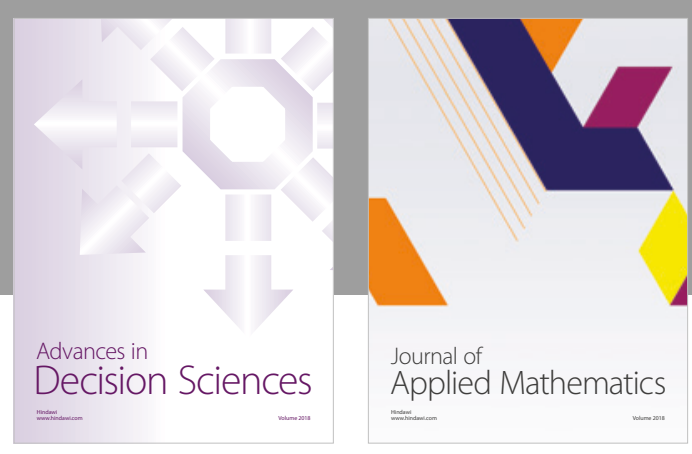

Journal of

Applied Mathematics
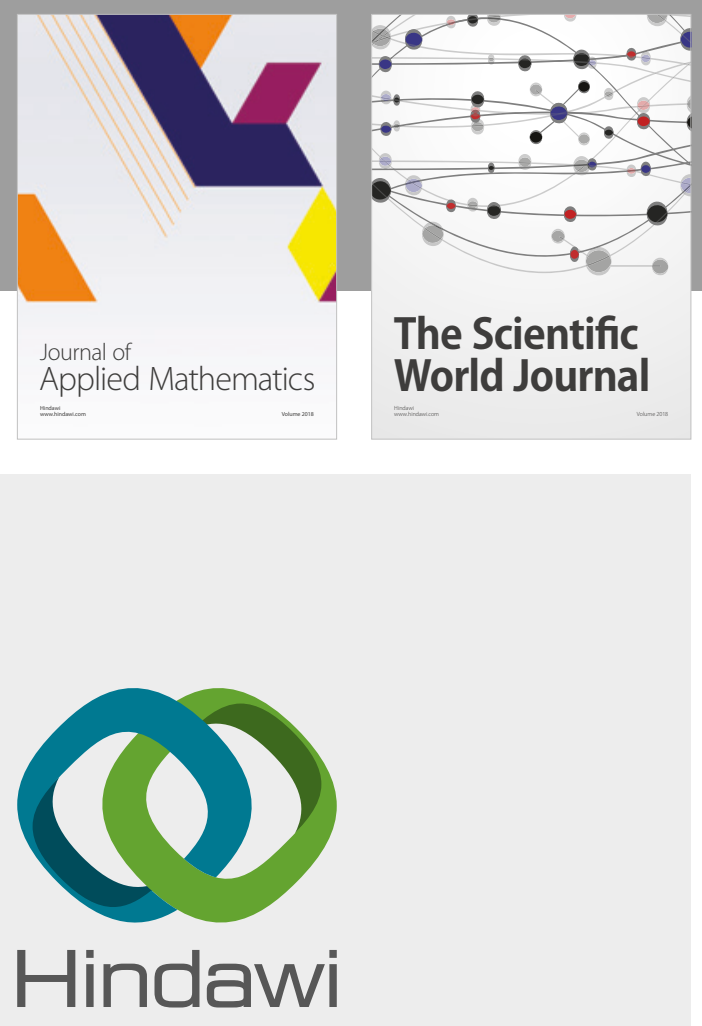

Submit your manuscripts at

www.hindawi.com

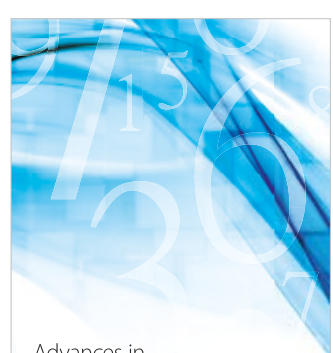

Advances in
Numerical Analysis
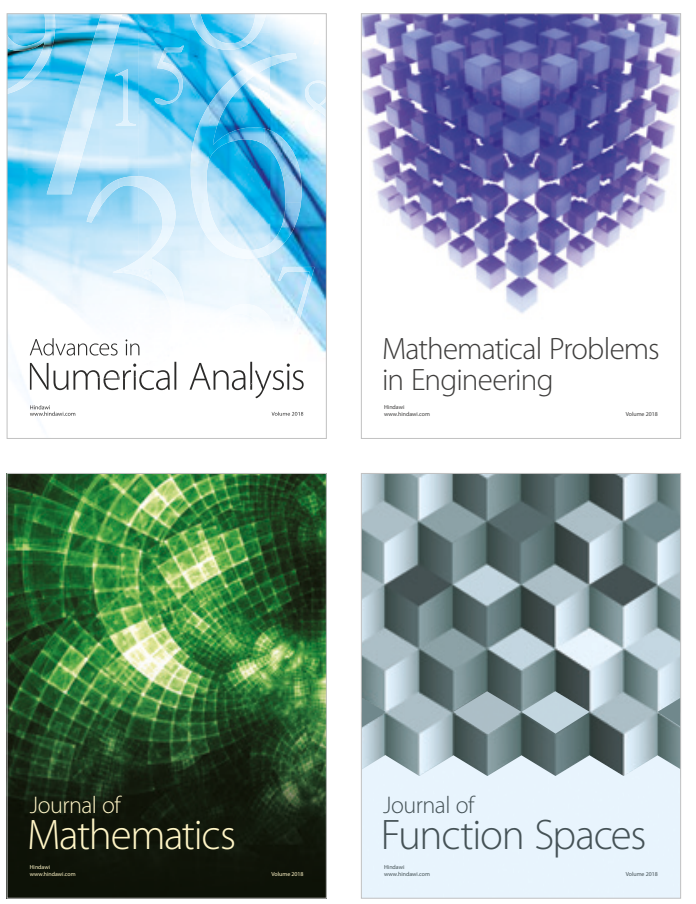

Mathematical Problems in Engineering

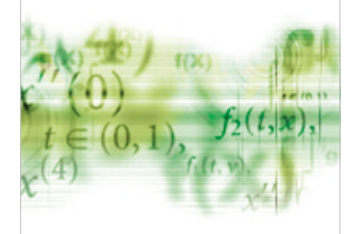

International Journal of

Differential Equations

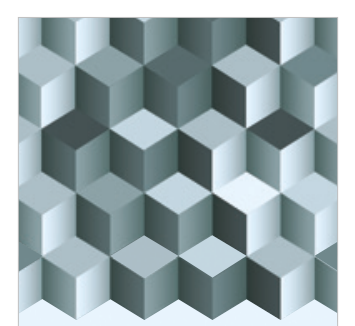

Journal of

Function Spaces

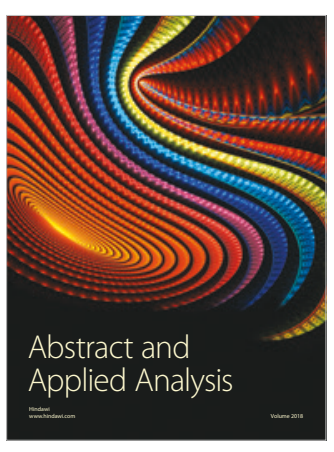

The Scientific

World Journal

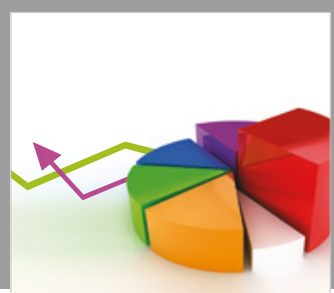

Journal of

Probability and Statistics
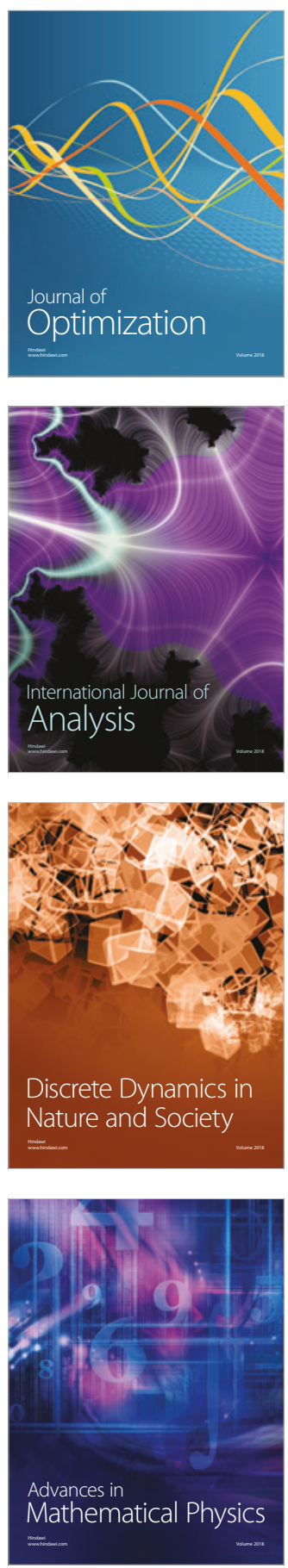